Background This preliminary audit aimed to assess the feasibility of referring children presenting to a UK District General Hospital Emergency Department (ED), seeing 27500 children per year, to their own Primary Care General Practitioner (GP) following an initial assessment in the ED.

Method One hundred and fifty-two patients were assessed by a Consultant in Paediatric Emergency Medicine in the ED during the triage process. Those fulfilling the audit inclusion criteria were referred directly from triage to their own off-site Primary Care GP for a same-day assessment.

Results Only ten patients (6.6\%) fulfilled the inclusion criteria for referral to Primary Care and seven (70\%) of these were accepted, none of whom were referred back to the hospital acutely by their GP. The median time spent in the ED for the 152 patients assessed in the audit was 1 hour 12 minutes and for the 10 patients referred to Primary Care was 31 minutes.

Conclusions Only a small number of children assessed in the audit were suitable for referral to Primary Care. The assessment and referral process was not a good use of Emergency Department resources.

The absence of a valid and reliable screening tool or early warning score to predict the safe discharge of children from an ED reduced the number of children that could be referred directly to primary care from the ED.

Further multi-centre work is required to evaluate a clinical decision-making framework to enable the accurate assessment of children for their safe discharge or referral from an ED.

\section{CHILD ABUSE DETECTION AT THE EMERGENCY DEPARTMENT USING A NEW PROTOCOL BASED ON PARENTAL CHARACTERISTICS}

doi:10.1136/archdischild-2012-302724.1480

H Diderich. Emergency Department, Medisch Centrum Haaglanden, The Haque, The Netherlands

Background and Aims The number of children identified as victims of child abuse in the Emergency Department represent only the tip of the iceberg. The "Hague-protocol" takes a new and successful approach to the recognition of child abuse at the E.D.

Methods These new guidelines call for notification to the Reporting Center for Child Abuse and Neglect when an adult patient who is responsible for children at home, visits the E.D. because of either 1) Substance abuse 2) Suicide attempt 3) Domestic violence. A before and after study was done at E.D.'s of five hospitals in an urban region in the Netherlands.

Results In the two years prior to the introduction of the protocol, a total of 4 parents were reported. After the guidelines were issued in 2007 the number of suspected child abuse cases reported to the Reporting Center, based on parental characteristics, rose substantially. A total number of 107 cases were reported in 2008, 149 cases in 2009, and 126 cases in 2010. Results indicate that child abuse was confirmed in the large majority (92\%) of the reported cases. In $5 \%$ of the cases child abuse could not be confirmed and in $3 \%$ of the cases it was concluded that there was no child abuse.

Conclusion The Hague-protocol can substantially improve and increase the detection rate of child abuse cases via the E.D. Parental characteristics can be strong predictors of child abuse, and can be included in E.D.'s guidelines to help identify new cases of child abuse.

\section{TRENDS IN PAEDIATRIC ACUTE ASTHMA MANAGEMENT IN THE REPUBLIC OF IRELAND: A COMPARISON BETWEEN COMMUNITY AND HOSPITAL PRACTICE}

doi:10.1136/archdischild-2012-302724.1481
1,2J Cronin, ' $\mathrm{J}$ Pollock, ${ }^{3} \mathrm{~T}$ Grant, ${ }^{1,2} \mathrm{~S}$ McCoy, ${ }^{4} \mathrm{~N}$ Breen, 'S Walsh, ${ }^{1,25} \mathrm{R}$ O'Sullivan. ${ }^{1}$ Paediatric Emergency Research Unit; ${ }^{2}$ National Children's Research Centre, Our Lady's Children's Hospital Crumlin; ${ }^{3}$ Centre for Support and Training in Analysis and Research (CSTAR); ${ }^{4}$ Department of General Practice; ${ }^{5}$ Department of Paediatrics, University College Dublin, Dublin, Ireland

Introduction The prevalence of childhood asthma in Ireland is amongst the highest in the world. We aim to compare the management of this condition by Emergency Physicians (EPs), Paediatricians and community-based General Practitioners (GPs).

Methods A standardised online anonymous survey was conducted of senior EPs, Paediatricians and General Practitioners (GPs) in Ireland. $15 \%$ of GPs nationally were chosen using a computerised randomization method. A postal survey was sent to those GPs for whom an email address was not available.

Results We received 344 responses, giving an overall response rate of $52.3 \%$ (EP: $67.6 \%$; Paediatricians: $51.3 \%$; GP: 50.6\%).

Over $70 \%$ of paediatricians and EPs use spacer devices for mild and moderate episodes; 78\% of GPs use spacers for mild asthma, however this drops to $38 \%$ for moderate episodes. Paediatricians and EPs differed in their choice of first-line intravenous bronchodilators with paediatricians choosing salbutamol (48.3\%) most frequently and EPs choosing magnesium (48.3\%). Whereas almost all respondents felt that severe asthma should be referred to an ED, over $85 \%$ of paediatricians and EPs felt that moderate attacks should be referred compared to $13.7 \%$ of GPs. Over $90 \%$ of all respondents would welcome a national guideline on the management of acute paediatric asthma exacerbations.

Conclusion This is the first study of its kind internationally to compare management of this important and common condition across the relevant specialties. The results of this survey support the development of a national guideline for acute paediatric asthma management.

\section{PAEDIATRIC TRAUMA TRANSFERS: ARE WE FAST ENOUGH?}

doi:10.1136/archdischild-2012-302724.1482

N Cooper, K Parkins, D Grainger. North West and North UK Paediatric Transport Service, Central Manchester Foundation NHS Trust, Warrington, UK

Background Children with traumatic brain injury (TBI) require rapid stabilisation and transfer to a paediatric multi-trauma centre (pMTC) within 3 hours (4 hours if rural) of injury (UK guidelines 2010). In our region transfer of children is either by local hospital team or regional paediatric transport service (NWTS started November 2010).

Methods Retrospective 16 month audit patients with TBI transferred into 2 regional pMTCs, identifying severity of injury, areas of delay, length of stay (LOS) on PIC and survival to hospital discharge.

Results 56 patients with TBI were identified. Median time of arrival at pMTC 355 minutes (IQR260-495), 210 minutes for time critical injuries (IOR180-270).

Areas of delay identified:

- Referral time post injury - median 115 minutes (IOR90-172)

- Insertion of invasive lines e.g. arterial or central in 54\%

- Neurosurgical Intervention at adult MTC in 4 patients

- Local team vs NWTS= 210 vs 431 minutes

Patients transferred by NWTS were more likely to have severe head injury and multi-trauma. However, median LOS was similar (local team 3.5 (IOR 2-10) vs NWTS 3 (IOR 1-6) days). Overall mortality rate was $6 \%$ (national mortality $=10 \%$ )

Conclusions Transfer children with TBI within 3-4 hour national target remains challenging. However, regional LOS and mortality rate remain low. This audit highlights the need for early referral, in line with the recent Trauma Network guidelines. The use of 